\title{
Standards müssen angewendet werden
}

\author{
Gewerkschaften messen der gesellschaftlichen \\ Verantwortung der Unternehmen sehr große \\ Bedeutung bei. Grundlage dieser Verantwor- \\ tung ist die verbindliche und überprüfbare \\ Erfüllung von Sozialstandards der Interna- \\ tionalen Arbeitsorganisation. \\ Von Uwe Wötzel
}

nationale Bund freier Gewerkschaften in der CSR-Debatte die Erfordernisse dieser Zeit.

Das wesentliche Problem im gegenwärtigen Prozess der Liberalisierung der Märkte sind die asymmetrischen politischen Strukturen und Defizite in der Regulierung der Märkte. Die Weltwirtschaft wird einseitig von den kurzfristigen Renditeinteressen der globalen Finanzmärkte bestimmt. Treiber der Globalisierung sind die Investoren von transnationalen Konzernen mit ihren Tochterunternehmen. Unternehmen bestimmen durch ihre einflussreiche Lobbyarbeit maßgeblich die Standards für den Freihandel und den Schutz von Investitionen. Ihr Interesse an einem gesellschaftlich verantwortlichen Handeln wächst mangels Pflicht bisher nur mit den Anforderungen von wenigen aufgeklärten Investoren. Erst durch sozialkritische Kampagnen von Gewerkschaften und NGOs wie etwa der Kampagne für saubere Kleidung ist die Vermeidung von Reputationsschäden ein starkes Motiv für die Anerkennung von Sozialstandards geworden. Viele Staaten verfolgen die Durchsetzung der Kernarbeitsnormen der International Labour Organisation (ILO) und ihres Arbeitsrechtes nicht mit dem notwendigen Engagement. Für dieses Engagement sind leistungsstarke demokratische Regierungen erforderlich, die jeden Unterbietungswettbewerb um niedrigere Steuern und minimale Sozialstandards beenden.

\section{Unternehmen und Transparenz}

Die Öffentlichkeit muss erkennen können, wie Unternehmen in den jeweiligen Stufen, Phasen und Orten der Wertschöpfungskette soziale und ökologische Mindeststandards erfüllen. Mindeststandards sind aus gewerkschaftlicher Sicht die Menschenrechte und die Sozialstandards der ILO. Diese Mindeststandards müssen schrittweise, dauerhaft und weltweit in den Unternehmen und bei ihren Vertragspartnern integriert werden. Dabei geht es nicht um eine Option, sondern um eine universelle Pflicht zur Förderung der Menschenrechte und der Sozialstandards, die sich aus der aktuellen Sachlage ableitet:

- Seit 1945 wird der Weltfrieden durch 300 Kriege und bewaffnete Konflikte gefährdet. Sozialer Unfrieden ist eine Hauptursache für diese Konflikte.

- Die durch Menschen verursachte Schädigung der Erdatmosphäre gefährdet die Lebensbedingungen von vielen Millionen Menschen.

- Armut, Unterernährung, fehlende Versorgung mit Trinkwasser, Gesundheits- und Bildungseinrichtungen bestimmen die Lebensbedingungen von über drei Milliarden Menschen. $\quad \rightarrow$ 


\section{„Die Arbeitsbedingungen in den Weltmarken-Fabriken der multinationalen Unternehmen und ihrer Zulieferer sind für die meisten Beschäftigten alles andere als attraktiv."}

- In den Zulieferketten der transnationalen Unternehmen wird durch die Verweigerung von angemessener Entlohnung Armut erzeugt und zugleich an dieser verdient.

- Politische Verfolgung ist mit Gefahren für Leib und Leben von Gewerkschaftern in sehr vielen Ländern an der Tagesordnung. Im Jahr 2003 wurden in 133 Staaten Gewerkschaftsrechte verletzt. Es werden Gewerkschafter getötet, verletzt, verhaftet und zu Haftstrafen verurteilt.

I Zwangsarbeit existiert noch in einigen Ländern.

- Durch Kinderarbeit wurden nach Schätzungen der ILO etwa 246 Millionen Kinder unter 15 Jahren ausgebeutet, davon über 70 Prozent in der Landwirtschaft und zwei Drittel mit gefährlichen Tätigkeiten.

- Diskriminierungen im Arbeitsalltag erfolgen in vielen Ländern aufgrund von Zugehörigkeit zu Geschlecht, sexueller Orientierung, Religion, Hautfarbe, Nationalität und politischer Meinung.

Die Arbeitsbedingungen in den Weltmarken-Fabriken der multinationalen Unternehmen und ihrer Zulieferer sind für die meisten Beschäftigten alles andere als attraktiv. Überlange Arbeitszeiten, Arbeit auf Abruf, unsoziale Schichtarbeitssysteme, Nachtarbeit, unfreiwillige Überstunden, schlechte und unpünktliche Bezahlung, wenig Respekt für gewerkschaftliche Rechte, starker gesundheitlicher Verschleiß, Belästigung durch schlechte Luft, ungeschützter Umgang mit Gefahrstoffen, sexuelle Belästigung am Arbeitsplatz, Unterdrückung gewerkschaftlicher Betätigung und Diskriminierungen unterschiedlichster Art gehören vielerorts zum Arbeitsalltag. Massenarbeitslosigkeit ist fast überall gegenwärtig.

Gewerkschaften wirken für die Verbesserung der sozialen Lage ihrer Mitglieder. Sozialer Schutz und menschenwürdige Arbeit müssen auf staatlicher und zwischenstaatlicher Ebene auf der Grundlage der Übereinkommen der Internationalen Arbeitsorganisation verbindlich geregelt werden. Nicht in allen Ländern existieren politische Strukturen, die eine Ratifizierung der ILO-Normen durchsetzen. Deshalb hat die ILO die „Erklärung über die grundlegenden Prinzipien und Rechte bei der Arbeit" angenommen. Damit bekennen sich die 178 ILO-Mitgliedstaaten ausdrücklich zu folgenden Grundprinzipien:
I Freiheit von Zwangsarbeit,

I Diskriminierungsfreiheit,

I Verbot von Kinderarbeit,

I Vereinigungsfreiheit und Recht auf Tarifverhandlungen.

Weitere grundlegende Arbeitsrechte werden vom Internationalen Bund freier Gewerkschaften (IBFG) seit 1997 gefordert:

I Zahlung existenzsichernder Löhne;

- keine überlangen Arbeitszeiten, das heißt nicht mehr als 48 Stunden pro Woche und mindestens ein freier Tag pro Woche. Nicht mehr als 12 Überstunden pro Woche und nur auf freiwilliger Basis und bei zusätzlicher Bezahlung;

I menschenwürdige Arbeitsbedingungen: Gesundheits- und Sicherheitsschutz am Arbeitsplatz, Verbot körperlicher Misshandlung, sexueller Belästigung und Einschüchterung sowie

I ein festes Beschäftigungsverhältnis mit arbeits- und sozialrechtlichen Verpflichtungen.

\section{OECD-Leitsätze - ein erster Maßstab}

Die OECD-Leitsätze für multinationale Unternehmen stellen den einzigen multilateral anerkannten und umfassendsten Kodex für Unternehmen dar, den Regierungen vereinbart haben (2). OECD-Leitsätze unterscheiden sich dadurch von den Kodizes, die sich Unternehmen selbst geben oder mit Gewerkschaften ausgehandelt haben. Die hier vereinbarten Prinzipien und Standards beziehen sich auf Arbeit und Beschäftigung, auf die Beziehungen zwischen den Sozialpartnern, Menschenrechte, Umwelt, Offenlegung von Informationen, Bekämpfung von Korruption, Wettbewerb, Verbraucherinteressen, Wissenschaft und Technologie sowie Besteuerung. Die Leitsätze enthalten seit dem Jahr 2000 zusätzliche Ausführungen zur Beseitigung von Kinder- und Zwangsarbeit und erfassen somit alle international anerkannten Kernarbeitsnormen. Im Umweltbereich werden die Unternehmen aufgefordert, ihre Umweltleistungen zu verbessern, Umweltmanagementsysteme einzuführen, die Umwelteffekte zu verringern und die entsprechende Berichterstattung zu verbessern. Im Rahmen der allgemeinen Grundsätze werden ferner Verantwortlichkeiten für die Zulieferfirmen und Unterauftragnehmer formuliert, die zur Anwendung der Prinzipien der OECD-Leitsätze veranlasst werden sollen.

Von besonderer Bedeutung sind die OECD-Leitsätze auch deshalb, weil sie sich auf die Länder beziehen, die weltweit die umfangreichsten Auslandsinvestitionen bereitstellen und auch die wichtigsten Empfänger ausländischer Direktinvestitionen sind.

Ohne Beteiligung der Beschäftigten und ihrer Gewerkschaften führen Maßnahmen unter dem Schirm von Corporate Social Responsibility nicht zu einer sozialen Teilhabe und verfehlen damit ihr eigentliches Ziel. Gewerkschaften sind notwendige Partner im Dialog und bei Kollektivverträgen. Gewerkschaften übernehmen auch eine wichtige Funktion bei Formulierung, Implementierung und Kontrolle, aber auch bei der Bekanntmachung von Arbeitnehmerrechten, Verhaltenskodi- 
zes und internationalen Rahmenabkommen. Der Internationale Bund Freier Gewerkschaften (IBFG) und die Global Unions haben durch die Publikation des „Basic Code of Labour Practice“ bereits 1997 einen Vorschlag für Mindestanforderungen an Verhaltenskodizes vorgelegt (3). Von zentraler Bedeutung sind der spezifische Einblick, die fachliche Kompetenz und die Erfahrung von Gewerkschaften mit Arbeitsproblemen in verschiedenen Branchen sehr vieler Länder. Gewerkschaften sind in vielen Betrieben präsent und genießen das Vertrauen der Arbeiterschaft. Sie können deshalb eine wichtige Funktion bei der Umsetzung und Kontrolle der Standards wahrnehmen. Sie wirken in Global Unions und treffen mit transnationalen Unternehmen internationale Rahmenvereinbarungen über die Durchsetzung von Sozialstandards (4). Aufgrund ihrer europäischen beziehungsweise internationalen Strukturen sind Gewerkschaften ein wichtiger Ansprechpartner, um Verhaltenskodizes und internationale Rahmenabkommen unternehmensspezifisch zu erarbeiten und verlässlich umzusetzen.

\section{Neue Instrumente}

Zwischen den Anforderungen der international geltenden Kernarbeitsnormen und der sozialen Realität besteht in den meisten Ländern eine große Kluft. Diese Kluft müssen alle Akteure durch vielfältige Anstrengungen schrittweise verkleinern. Selbstverständlich tragen die jeweiligen Regierungen für die Umsetzung internationaler Normen die Hauptverantwortung. Da die Bemühungen der Regierungen bisher nicht ausreichen, sind neue Anstrengungen erforderlich.

Diese müssen der Entwicklung von wirksamen Verfahren, Aufsichtsbehörden, Beschwerde- und Klagesystemen und dem Aufbau unabhängiger Gewerkschaften dienen. Wichtig für die Einhaltung der Sozialstandards in Ländern mit schwachen Aufsichtsbehörden sind regelmäßige externe Überprüfungen der Einhaltung von Sozialstandards durch unabhängige Institutionen, die mit lokalen Akteuren wie Gewerkschaften und Nichtregierungsorganisationen zusammenarbeiten. Private Richtlinien der ISO können weder Normen neu definieren noch bestehende interpretieren (5).

Das Europäische Parlament (EP) verlangt legislative Maßnahmen zur Förderung der sozialen Verantwortung der Unternehmen. Bereits im Mai 2002 forderte das EP in dem Mandat der EU-Kommission für den Weltgipfel für nachhaltige Entwicklung in Johannesburg „einen Rechtsrahmen für die soziale Verantwortung der Unternehmen in privaten EU-Kapitalgesellschaften bis 2004, der von privaten Investoren verlangt, die grundlegenden Arbeitnehmerrechte und Umweltnormen zu beachten, die in den einzelstaatlichen und internationalen Rechtsvorschriften festgelegt sind“. Dem Willen des EP muss nun der Europäische Rat folgen.

Ein weiterer Anknüpfungspunkt sind die von der UN-Unterkommission zum Schutz und zur Förderung der Menschenrechte einstimmig angenommenen „Normen der Vereinten Nationen für die Verantwortlichkeit transnationaler Unternehmen im Hinblick auf die Menschenrechte“. Der Vorschlag fordert die Staaten unter anderem auf, durch Instrumente wie Entschädigungspflichten, Inspektionen oder Ombudspersonen die staatliche Verantwortung zum Schutz der Menschen wahrzunehmen und gegenüber den Unternehmen durchzusetzen.

Für die Durchsetzung der Vorschläge brauchen wir starke demokratische und leistungsfähige Staaten. Sie sollten folgenden Vorschlägen folgen:

I Unternehmen müssen im Rahmen ihrer Bilanzen eine Rechenschaftspflicht über die Einhaltung von Umwelt- und Sozialstandards in ihren Wertschöpfungsprozessen erfüllen. In der gesamten Lieferantenkette sind die Sozialstandards zu integrieren. Durch ein unabhängiges Monitoring ist die Einhaltung der Standards zu überprüfen.

I Kapitalgesellschaften erhalten Börsenzulassung, Kredite und Außenhandelsbürgschaften nur, wenn sie die Regeln dieser Rechenschaftspflicht erfüllen.

- Unternehmen erhalten Aufträge und Beihilfen der öffentlichen Hand nur, wenn sie die Kriterien dieser Rechenschaftspflicht erfüllen.

I Die EU macht bestehende Sozial- und Umweltstandards zum festen Bestandteil ihrer Außenhandelspolitik und setzt diese in der Welthandelsorganisation durch.

- Jede Bürgerin und jeder Bürger erhält das Recht auf kostenlosen und leichten Zugang zu überprüfbaren Informationen über das soziale und ökologische Verhalten von Unternehmen.

I Informanten („whistle blower“) von Berichtspflichtverletzungen erhalten umfassenden Schutz.

I Gesellschaftliche Unternehmensverantwortung wird Lernziel und Thema in der allgemeinen und in der beruflichen Aus- und Weiterbildung.

\section{Anmerkungen}

(1) Alle Zitate bis dahin unter http://congress.icftu.org/ displaydocument.asp? Index=991220349\&Language=DE

(2) www.tuac.org/publicat/Guidelines-german.pdf

(3) www.global-unions.org sowie www.itcilo.it/english/actrav/telearn/global/ilo/guide/icftuco.htm

(4) www.imfmetal.org/main/index.cfm? $\mathrm{n}=47 \& \mathrm{l}=2 \& \mathrm{c}=10266$

(5) vgl. dazu IBFG-Resolution vom Dezember 2004, http://congress.icftu.org/ displaydocument.asp? Index=991220349\&Language=DE
- AUTOR + KONTAKT

Uwe Wötzel ist Referatsleiter im Bereich Politik und Planung der Vereinten Dienstleistungsgewerkschaft (ver.di), Bundesverwaltung Berlin. ver.di, Ressort 1, Paula-Thiede-Ufer 10,

10179 Berlin. Tel. 030/6956-1036, E-Mail: uwe.woetzel@verdi.de 
(c) 20I0 Authors; licensee IÖW and oekom verlag. This is an article distributed under the terms of the Creative Commons Attribution Non-Commercial No Derivates License (http://creativecommons.org/licenses/by-nc-nd/3.o/), which permits unrestricted use, distribution, and reproduction in any medium, provided the original work is properly cited. 